\title{
Impact of major video-assisted thoracoscopic surgery on care quality
}

\author{
Florencio Quero Valenzuela ${ }^{1}$, Inmaculada Piedra Fernández ${ }^{1}$, Maria Del Carmen Martínez Cirre ${ }^{2}$, Abel \\ Sánchez-Palencia ${ }^{1}$, Antonio Cueto Ladrón de Guevara ${ }^{1}$
}

${ }^{1}$ Section of Thoracic Surgery, Hospital Universitario Virgen de las Nieves de Granada, Granada, Spain; ${ }^{2}$ Hospital Clinical Documentation Service, Hospital Universitario Virgen de las Nieves de Granada, Granada, Spain

Contributions: (I) Conception and design: F Quero Valenzuela, I Piedra Fernández, A Cueto Ladrón De Guevara; (II) Administrative support: F Quero Valenzuela, I Piedra Fernández, M Del Carmen Martínez Cirre; (III) Provision of study materials or patients: F Quero Valenzuela, I Piedra Fernández, M Del Carmen Martínez Cirre, A Cueto Ladrón de Guevara; (IV) Collection and assembly of data: F Quero Valenzuela, I Piedra Fernández, M Del Carmen Martínez Cirre, A Sánchez-Palencia, A Cueto Ladrón de Guevara; (V) Data analysis and interpretation: F Quero Valenzuela, I Piedra Fernández, M Del Carmen Martínez Cirre; (VI) Manuscript writing: All authors; (VII) Final approval of manuscript: All authors. Correspondence to: Florencio Quero Valenzuela, MD. Section of Thoracic Surgery, Hospital Universitario Virgen de las Nieves de Granada, Avda de las Armadas s/n 18001, Granada, Spain. Email: florencioquero@msn.com.

Background: The objective of this study was to investigate the impact of a program of major video-assisted surgery on care quality in a Unit of Thoracic Surgery.

Methods: A descriptive comparative study was conducted of 793 major thoracic procedures performed between 2009 and 2012. Quality indicators and hospital performance before [2009-2010] and after (2011 and 2012) the implementation of the program.

Results: The incidence of surgical complications decreased significantly from $6.32 \% / 7.88 \%(2009 / 2010$, respectively) to $1.87 \% / 1.67 \%$ (2011/2012, respectively) [95\% CI for 7.08\% (4.20-9.96\%); 95\% CI for $1.76 \%$ (0.44-3.08\%) $\mathrm{P}<0.001$, respectively]. The mean hospital stay was reduced from $8.5 / 7.8$ days in $2009 / 2010$, respectively, to $6.3 / 5.8$ days in $2011 / 2012$, respectively. Mortality rates were $0.57 \%, 0.60 \%, 0.93 \%$ and $0.43 \%$ in 2009, 2010, 2011, and 2012, respectively ( $\mathrm{P}=0.624,95 \% \mathrm{CI}:-0.6,0.7)$. The percentages of emergency readmissions in 2009/2010 were $1.16 \% / 1.23 \%$, respectively vs. $2.80 \% / 0.84 \%$ in $2011 / 2012$.

Conclusions: The implementation of the video-assisted thoracic surgery (VATS) program in the unit of Thoracic Surgery Care resulted in a significant improvement in care quality, with a reduction of length of hospital stay, but without any changes in mortality or the percentage of readmissions at 30 post-operative days.

Keywords: Quality care; video-assisted thoracic surgery (VATS); mean stay (MS); surgical complications; mortality

Submitted Jun 18, 2017. Accepted for publication Sep 28, 2017.

doi: $10.21037 /$ jtd.2017.10.13

View this article at: http://dx.doi.org/10.21037/jtd.2017.10.13

\section{Introduction}

Care quality is essential for an efficient management of thoracic surgery units. It is strengthened by evidence-based practice operated within a framework of safety, efficacy and effectiveness $(1,2)$. Another aspect related to care quality is outcome assessment. Care quality can be evaluated by different instruments including clinical databases, clinical data sources, and audits, among other. Data sources of our hospital include a control panel of healthcare activity and a Basic Minimum Set of Data (BMSD).

Recent research studies consistently support the advantages of video-assisted thoracic surgery (VATS), especially in lung cancer. VATS reduces post-operative pain, lung function impairment, inflammatory response, hospital stay, and the occurrence of post-surgical complications (3-5). 
It Ii important that the outcomes of enhanced surgery programs and new surgical techniques are evaluated.

With the purpose of improving care quality and incorporating new scientific-technological advances, the Clinical Unit of Thoracic Surgery of the University Hospital Complex of Granada, Spain, incorporated a minimallyinvasive thoracic surgery program for major thoracic surgical procedures in January 2011. To carry out the thoracic surgery unit he incorporated a surgeon with extensive experience in thoracoscopic surgery that led the implementation of the program. The training of surgeons was performed with training in the hospital experimental surgery unit and the support of the consulting surgeon. Two years after the implementation of the program, an assessment was performed of improvements achieved in care quality.

The primary objective of this study was to determine if care quality improved with the implementation of a major video-assisted surgery program incorporated in our Unit of Thoracic Surgery in 2011.

\section{Methods}

A descriptive, comparative study of surgery outcomes before and after the implementation of a VATS program was performed using care quality and hospital performance indicators.

Outcomes were extracted from hospital data sources. The BMSD provides a generic set of influence variables related to disease, diagnosis, patient, and hospital performance. We compared quality indicators and hospital performance before [2009-2010] and after [2011-2012] the implementation of the program.

Data were collected by the Clinical Documentation Unit of our hospital and reports were prepared by IASIST S.A. (6) for the University Hospital Complex of Granada, which belongs to the Andalusian Healthcare System (SSPA). Data were encoded by medical specialists in clinical documentation based on discharge reports according to the International Classification of Diseases, Version 9, Clinical Modification (ICD-9-CM). No modifications were made to the encoding system by the Clinical Documentation Unit during the study period [2009-2012].

Case identification was based on a search for the GRD 538 and GRD 075 codes, which refer to major thoracic procedures with or without major complications. The cases were detected using the ICD-9-CM coding system. Thoracotomy pulmonary lobectomy was identified with the ICD-9-CM codes 32.3 and 32.4; codes 32.5 and 32.6 were used for pneumonectomy. Video-assisted thoracic procedures were identified using codes 32.30 for thoracoscopic segmental resection of the lung, 32.41 for thoracoscopic lung lobectomy, and 32.50 for thoracoscopic pneumonectomy. Codes 7.81, 7.84 for open or thoracoscopic thymectomy respectively and code 34.3 for mediastinal tumors (7).

\section{Quality and hospital performance indicators}

Study indicators and outcomes were evaluated according to the definitions and calculations established in the SSPA BMSD system (8) and include: percentage of complications, percentage of urgent readmissions at 30 days after discharge; mortality rate and standardized mortality ratio (SMR); mean stay (MS) in the clinical unit; MS of patients undergoing pulmonary lobectomy; impact on the stay that is an indicator resulting from the product of the difference from the standard by the number of cases; case-mix index and relative weight.

The percentage of complications was calculated based on total discharges related to the same surgical group after scheduled or emergency admission. Postoperative complications included urinary tract, respiratory, cardiovascular, nervous, and gastrointestinal complications, septicemia and surgical wound infection.

MS was calculated as the mean number of days spent by patients in hospital until discharge. Readmission was defined as unexpected rehospitalization (emergency admission) within the first 30 days after discharge.

Overall mortality was defined as the number of discharges due to death. SMR was calculated as the ratio between the observed and the expected number of deaths, as estimated by the indirect standardization method (ratio between the mortality rate observed in the Service and the expected mortality rate for its series according to the standard IASIST: comparative with hospitals of national database).

Relative weight was defined as the resource use attributed to each diagnosis-related group and the average of the relative weight is calculated on all GRDs of the Service.

The case-mix index or weight was defined as the characteristics of patients, including disease severity, prognosis, difficulty of treatment, need for medical care and resource use. The case-mix index allows to determine if the cases treated in a hospital were more or less challenging than standard.

Other clinical features or surgical time of the 
Table 1 The type and number of procedures performed is displayed by year

\begin{tabular}{|c|c|c|c|c|}
\hline Procedures & Year 2009, n (\%) & Year 2010, n (\%) & Year 2011 (VATS program), n (\%) & Year 2012 (VATS program), n (\%) \\
\hline Pneumonectomy & $7[4]$ & $6(3.6)$ & $8(3.7)$ & $10(4.1)$ \\
\hline Lobectomy & $79(45.5)$ & $67(40.6)$ & $85(39.7)$ & $96(40.0)$ \\
\hline Sublobar resections & $80(46.0)$ & $86(52.2)$ & $109[51]$ & $121(50.5)$ \\
\hline $\begin{array}{l}\text { Thymectomy and } \\
\text { mediastinal tumors }\end{array}$ & $8(4.5)$ & $6(3.6)$ & $12(5.6)$ & $13(5.4)$ \\
\hline Total procedures & 174 & 165 & 214 & 240 \\
\hline
\end{tabular}

VATS, video-assisted thoracic surgery.

interventions were not available for this study.

\section{Study population}

The population of study was composed of patients undergoing major thoracic surgery at the Clinical Management Unit of Thoracic Surgery of the University Hospital Complex of Granada, Spain from 2009 to 2012. Surgical procedures included pulmonary resections (lobectomy, pneumonectomy, segmental and extended resections), mediastinal tumors and thymectomies. Candidates for surgery complied with the guidelines and recommendations of clinical practice guidelines for lung cancer and pulmonary metastasis $(9,10)$. Indications were not modified during the study period. After incorporating the program, patients were not randomized to receive either technique, but surgical decision-making was based on surgeon's judgement and the clinical characteristics or disease stage of the patient. Drainage removal was performed with a flow rate of less than $200 \mathrm{cc}$ and check for absence of air leakage and no changes were made to this practice during the study period.

The study was approved by the ethics committee of University Hospital Complex of Granada (CEI 080615). Written informed consent was obtained from all patients.

\section{Statistical analysis}

Statistical analysis was performed using the SPSS Statistics 17.0.1 package. A $\mathrm{P}$ value $<0.05$ was considered statistically significant, and $95 \%$ confidence intervals were calculated. Central tendency means, absolute frequencies, relative frequencies, proportions and rates were calculated. Analysis included the Chi-square test, Fisher's test, Welch two sample test when applicability criteria were met.

\section{Results}

The total number of major thoracic procedures performed during the study period was 793 . The percentage of pulmonary resections by video-assisted thoracoscopy was $43.45 \%$ in 2011 and $66.6 \%$ in 2012 . The type and number of procedures performed is displayed by year in Table 1 .

Case-mix indexes for 2009/2010 were 0.94/0.96, respectively, as compared to $0.94 / 1.02$ for $2011 / 2012$ $(\mathrm{P}=0.58,95 \% \mathrm{CI}:-0.43,0.37)$. The mean relative weight for $2009 / 2010$ was $0.95 / 1.04$, respectively $v s$. $0.98 / 1.02$ for $2011 / 2012$ ( $\mathrm{P}=0.14,95 \% \mathrm{CI}:-0.17,0.37)$. The characteristics of the patients; Age, gender, case mix index and relative mean weight are shown in Table 2.

Care quality indicators show a decrease in the percentage of surgical complications after the implementation of the program. Absolute and percentual values for surgical complications are shown in Table 3. The percentage of surgical complications dropped significantly between 2009/2010 (6.32\%/7.88\%, respectively) and 2011/2012 (1.87\%/1.67\%, respectively), $\mathrm{P}<0.00195 \% \mathrm{CI}$ for each proportion were $7.08 \%(4.20-9.96 \%)$ and $1.76 \%(0.44-3.08 \%)$.

Mortality was $1(0.57 \%), 1(0.60 \%), 2(0.93 \%)$ and $1(0.42 \%)$ in $2009,2010,2011$, and 2012, respectively $(\mathrm{P}=0.624,95 \%$ CI: $-0.6,0.7)$. SMRs for years $2009 / 2010$ were $0.25 / 0.45$, and $0.38 / 0.14$ for years $2011 / 2012$. Mortality values are shown in Table 4. The proportion of emergency readmissions 30 days after discharge was $1.16 \% / 1.23 \%$ in $2009 / 2010$, respectively vs. $2.80 \% / 0.84 \%$ in 2011/2012, respectively.

Hospital performance indicators demonstrate a decrease of MS in the Unit from 8.5/7.8 days in 2009/2010, respectively, to $6.3 / 5.8$ days in $2011 / 2012$, respectively. Comparative analysis of annual MS vs. standard stay is displayed in Table 5. The shortening of MS in 2011 and 2012 resulted in a -260 and -93 spare of stays with 
Table 2 The characteristics of the patients

\begin{tabular}{lccccc}
\hline Characteristics & Year 2009 & Year 2010 & Year 2011 (VATS program) & Year 2012 (VATS program) & P value \\
\hline Age mean (SD), years & $61(10.4)$ & $66(8.6)$ & $63(10.3)$ & $0.5)$ & 0.12 \\
Gender & & & & $48(20.5 \%)$ \\
Female & $36(20.1 \%)$ & $39(23.6 \%)$ & $45(21.0 \%)$ & $121(79.5 \%)$ \\
Male & $138(79.9 \%)$ & $126(76.4 \%)$ & $169(79.0 \%)$ & 1.02 & 1.02 \\
Case-mix index & 0.94 & 0.96 & 0.94 & 0.98 & 240 \\
Mean relative weight & 0.95 & 1.04 & 214 & 0.14 \\
Total procedures & 174 & 165 & & - \\
\hline
\end{tabular}

VATS, video-assisted thoracic surgery.

Table 3 Surgical complications

\begin{tabular}{|c|c|c|c|c|c|}
\hline Surgical complications & Year 2009 & Year 2010 & Year 2011 (VATS program) & Year 2012 (VATS program) & $P$ value \\
\hline Cardiovascular & 3 & 3 & 2 & 1 & - \\
\hline Urinary & 1 & 1 & 0 & 0 & - \\
\hline Surgical wound infection & 0 & 1 & 0 & 0 & - \\
\hline Value & 11 & 13 & 4 & 4 & $<0.001$ \\
\hline Percentage (\%) & 6.32 & 7.88 & 1.87 & 1.67 & - \\
\hline Total & 174 & 165 & 214 & 240 & - \\
\hline
\end{tabular}

VATS, video-assisted thoracic surgery.

Table 4 Annual evolution of mortality

\begin{tabular}{lcccc}
\hline Mortality & Year 2009 & Year 2010 & Year 2011 & Year 2012 \\
\hline Value & 1 & 1 & 214 & 240 \\
Total procedures & 174 & 165 & 0.93 & 0.42 \\
Mortality rate (\%) & 0.57 & 0.60 & 0.38 & 0.14 \\
SMR & 0.25 & 0.45 & & 2 \\
\hline
\end{tabular}

SMR, standardized mortality ratio.

respect to 2009/2010 (179/172, respectively). The MSs for thoracotomy lobectomy procedures performed in $2009 / 2010$ were $7.55 / 7.36$ days, respectively, as compared to $3.26 / 3.46$ for the same procedure in $2011 / 2012$.

\section{Discussion}

Quality indicators have been proven to be reliable when evaluating care quality and the effectiveness of improvement measures. Also, these indicators facilitate follow-up and comparison of the most relevant aspects related to medical practice (11-14).

Care quality improved significantly with the incorporation of the VATS program in the Unit of Thoracic Surgery, with a reduction of surgical complications. Care quality indicator values (percentage of complications, 
Table 5 Comparative analysis of annual mean stay vs. standard stay

\begin{tabular}{lcccc}
\hline Variables & Year 2009 & Year 2010 & Year 2011 (VATS program) & Year 2012 (VATS program) \\
\hline MS & 8.5 & 7.8 & 6.3 & 5.8 \\
MS standard & 8.6 & 7.7 & 7.1 & 5.9 \\
Difference & -0.1 & 0.1 & -0.8 & -0.1 \\
Impact of stays & 179 & 172 & -260 & -93 \\
\hline
\end{tabular}

VATS, video-assisted thoracic surgery; MS, mean stay.

mortality and readmission rates) were similar-although slightly higher- to those reported in a recent benchmarking study including 17 units of thoracic surgery in Spain (15).

The implementation of the VATS program resulted in a notable reduction in the percentage of surgical complications from $6-7 \%$ to $2 \%$, which is consistent with the results of previous studies (15). This significant reduction demonstrates that surgical outcomes have improved with the implementation of the VATS program in our Unit.

In contrast, mortality remained stable $(<1 \%)$, which is suggestive of the safety of the program. Reported mortality rates range from $0.2 \%$ to $3.1 \%$. However, variability in the percentage of pneumonectomies or extended surgical procedures performed has been witnessed among different centres complicating a cross-study comparison (15-18).

Case-mix indexes and relative weight did not change significantly during the study period. These indicators of hospital performance show that the type of surgical patients did not change significantly during the implementation of the program, which discards the possibility that the criteria for selecting candidates for thoracic surgery are more restrictive since the implementation of the program.

The learning curve of the surgeons did not affect the implementation of the VATS program possibly due to the training received. The degree of implementation was high and reached most patients undergoing major thoracic surgery after the second year.

The rate of readmissions or emergency readmissions within 30 days from discharge is a relevant indicator of care quality. Considerable differences are found in the values reported so far, with variations ranging from 0 to $9 \%$. Heterogeneity in terms of the type of resection and approach employed in each study hinders that reliable conclusions are drawn. Varela et al. (17) reported a rate of readmissions after major resections (lobectomy and pneumonectomy) of $6.9 \%$. Other studies $(19,20)$ included atypical VATS segmentectomies and resections, with an overall readmission rate of $8-9 \%$. Of note, the studies performed within the framework of the benchmarking study conducted in Spain from 2011 to 2015 documented readmission rates of 2.6$7.3 \%$ and $0-6.4 \%$ respectively $(15,18)$.

The impact of the type of VATS approach thoracotomy on the rate of readmissions has only been assessed in two previous studies $(19,20)$. The first study was favourable to conventional approaches, whereas the other reported no remarkable differences. The results of our study show that the rate of emergency readmissions after the implementation of the VATS program ranged from $0.8-2.8 \%$. These rates did not change significantly with the program or with the reduction of the MS. This could be explained by the fact that some patients may have been seen in other hospitals and not appear as cases of readmission. Yet, this possibility is contradicted by the fact that this type of patients is all referred from their hospitals to our unit. Further studies should be conducted to identify factors related to emergency readmissions in patients undergoing pulmonary resection.

The MS in the Unit of Thoracic Surgery decreased significantly since the implementation of the VATS program, with a remarkable number of hospital stays saved. The MS of patients undergoing the surgical procedures included in the VATS program decreased notably since it was first introduced two years ago. However, the improved care quality cannot be exclusively attributed to the VATS program. The reduction of hospital stays can be credited to the high proportion of VATS pulmonary resections included in the program during the first two years of implementation. As it was mentioned above, drainage withdrawal and discharge criteria did not change during the study. However, it should be noted that changes have been introduced in postoperative approach in our Unit in the last years, such as the introduction of a single chest tube and early withdrawal, which may have contributed to the 
reduction of the length of hospital stays.

The MSs for videothoracoscopic lobectomy were 3 and 3.4 days. This is consistent with most case series studies on thoracoscopic pulmonary resections $(4,5)$. However, there is wide variability in the MS for video-assisted lobectomy, which ranges from 3.7 to 12.6 days (15).

A limitation of this study may be the selection of the subjects included in the program. Yet, the inclusion criteria for videothoracoscopic surgery were established complying with the indications set forth in clinical practice guidelines.

Another limitation might be the quality of data recorded in clinical records. This possibility is countered by the fact that no changes were made to the electronic medical record software or in the coding system used in the Clinical Documentation Unit during the study period.

The ICD-9 MC coding system remained unaltered during the study period, which means that the percentage of missing data should be similar for the two groups, as they were encoded by the same specialists using the same system. Some authors argue that this type of databases is not reliable, as a loss of information occurs when patients are grouped by groups. However, BMSD provides the most relevant clinical variables such as complications, mortality or readmission rates (14).

The use of a BMSD in our study guarantees that data were not manipulated by study participants, as they were collected from the hospital BMSD. Additionally, the design of this study makes it possible to perform comparative studies of outcomes in other units or in different periods.

Despite its limitations, BMSDs are a useful instrument for evaluating care quality when other more precise clinical databases are not available. In conclusion, care quality in our unit improved with the implementation of the videoassisted major thoracic surgery program, resulting in a reduction in the mean hospital stay and the percentage of surgical complications.

\section{Acknowledgements}

Acknowledgements to the doctoral program of the University of Granada.

\section{Footnote}

Conflicts of Interest: The authors have no conflicts of interest to declare.
Ethical Statement: The study was approved by the ethics committee of University Hospital Complex of Granada (CEI 080615). Written informed consent was obtained from all patients.

\section{References}

1. Peiró S. The best hospitals. Between the need for comparative information and confusion. Rev Calid Asist 2001;16:119-30.

2. Peiró S, del Llano J, Queceddo L, et al. Diccionario de gestión sanitaria para médicos. Madrid: Fundación AbbotFundación Gaspar Casal, 2009.

3. McKenna RJ Jr, Houck W, Fuller CB. Video-assisted thoracic surgery lobectomy: experience with 1,100 cases. Ann Thorac Surg 2006;81:421-5; discussion 425-6.

4. Yim AP, Wan S, Lee TW, et al. VATS lobectomy reduces cytokine responses compared with conventional surgery. Ann Thorac Surg 2000;70:243-7.

5. Loscertales J, Quero Valenzuela F, Congregado M, et al. Video-assisted thoracic surgery lobectomy: results in lung cancer. J Thorac Dis 2010;2:29-35.

6. IASIST. Top 20 - 2000. Available online: http://www.iasist. com/es/1118/Codificacion

7. Statistical normalization. Available online: http://www. mspsi.gob.es/estadEstudios/portada/home.htm

8. CMBD Andalucía. Avaible online: http://www. juntadeandalucia.es/servicioandaluzdesalud/principal/ documentosacc.asp?pagina=profesionales_cmbd

9. Vansteenkiste J, De Ruysscher D, Eberhardt WE, et al. Early and locally advanced non-small-cell lung cancer (NSCLC): ESMO Clinical Practice Guidelines for diagnosis, treatment and follow-up. Ann Oncol 2013;24 Suppl 6:vi89-98.

10. Howington JA, Blum MG, Chang AC, et al. Treatment of stage I and II non-small cell lung cancer: Diagnosis and management of lung cancer, 3rd ed: American College of Chest Physicians evidence-based clinical practice guidelines. Chest 2013;143:e278S-e313S.

11. Rajaram R, Ju MH, Bilimoria KY, et al. National evaluation of hospital readmission after pulmonary resection. J Thorac Cardiovasc Surg 2015;150:1508-14.e2.

12. Medbery RL, Gillespie TW, Liu Y, et al. Socioeconomic Factors Are Associated With Readmission After Lobectomy for Early Stage Lung Cancer. Ann Thorac Surg 2016;102:1660-7. 
13. Wray NP, Ashton CM, Kuykendall DH, et al. Using administrative databases to evaluate the quality of medical care: a conceptual framework. Soc Sci Med 1995;40:1707-15.

14. Garnick DW, Hendricks AM, Comstock CB. Measuring quality of care: fundamental information from administrative datasets. Int J Qual Health Care 1994;6:163-77.

15. Freixinet Gilart J, Varela Simó G, Rodríguez Suárez P, et al. Benchmarking in Thoracic Surgery. Third Edition. Arch Bronconeumol 2016;52:204-10.

16. Varela G, Molins L, Astudillo J, et al. Pilot benchmarking experience in thoracic surgery: comparison of the casuistry and quality indicators in pulmonary resection. Arch Bronconeumol 2006;42:267-72.

Cite this article as: Quero Valenzuela F, Piedra Fernández I, Del Carmen Martínez Cirre M, Sánchez-Palencia A, Cueto Ladrón de Guevara A. Impact of major videoassisted thoracoscopic surgery on care quality. J Thorac Dis 2017;9(11):4454-4460. doi: 10.21037/jtd.2017.10.13
17. Varela G, Aranda JL, Jiménez MF, et al. Emergency hospital readmission after major lung resection: prevalence and related variables. Eur J Cardiothorac Surg 2004;26:494-7.

18. Freixinet JL, Varela G, Molins L, et al. Benchmarking in thoracic surgery. Eur J Cardiothorac Surg 2011;40:124-9.

19. Bravo Iñiguez CE, Armstrong KW, Cooper Z, et al. Thirty-Day Mortality After Lobectomy in Elderly Patients Eligible for Lung Cancer Screening. Ann Thorac Surg 2016;101:541-6.

20. Dickinson KJ, Taswell JB, Allen MS, et al. Unplanned Readmission After Lung Resection: Complete Follow-Up in a 1-Year Cohort With Identification of Associated Risk Factors. Ann Thorac Surg 2017;103:1084-91. 